\title{
Optical biopsy in real time by endocytoscopy: a case of juvenile polyp
}

Endocytoscopy allows ultra-high magnification, thereby enabling observation of structural and cellular atypia [1,2]. Additionally, endocytoscopy has been reported to have high diagnostic performance in differentiating neoplastic from nonneoplastic colorectal polyps, and can provide an alternative histological diagnosis to standard biopsy $[3,4]$.

A 44-year-old-man underwent colonoscopy at another hospital and a sessile polyp of remarkable redness was detected. Endoscopically the lesion was suspected to be early cancer and he was referred to our hospital.

White-light imaging revealed a reddish lesion with erosion in the rectum. The lesion was of the Is type, $15 \mathrm{~mm}$ in diameter ( Fig.1a). Chromoendoscopy showed elongated and irregularly arranged ducts ( Fig.1 b,c). A dense pattern was observed close to the dilated ducts by magnifying endoscopy with narrowband imaging ( $\triangleright$ Fig.1 d). Therefore, we suspected the lesion to be a hamartoma such as a juvenile polyp. Endocytoscopy (CF-Y0058-I; Olympus, Japan) showed elongated ducts with no apparent irregular nuclear findings around the ducts, and these features suggested that the lesion was nonneoplastic ( $\triangleright$ Video 1$)$. Ultimately, we diagnosed the lesion endoscopically as a juvenile polyp and endoscopic mucosal resection was performed. Histologically, edematous inflamed stroma and cystically dilated glands without atypia were seen ( $\mathbf{F i g . 2}$ ). Thus, the lesion was diagnosed as a juvenile polyp.

Juvenile polyp is known to be a smooth red mass projecting into the lumen of the colorectum. However, on diagnostic endoscopy it is sometimes difficult to differentiate juvenile polyp from cancer due to its form and structural features such as surface erosion and roughness [5]. Endocytoscopy, on the other hand, highlights the structure of nuclei and ducts, and therefore made it easy on this occasion to diagnose the nonneoplastic lesion with high confidence.
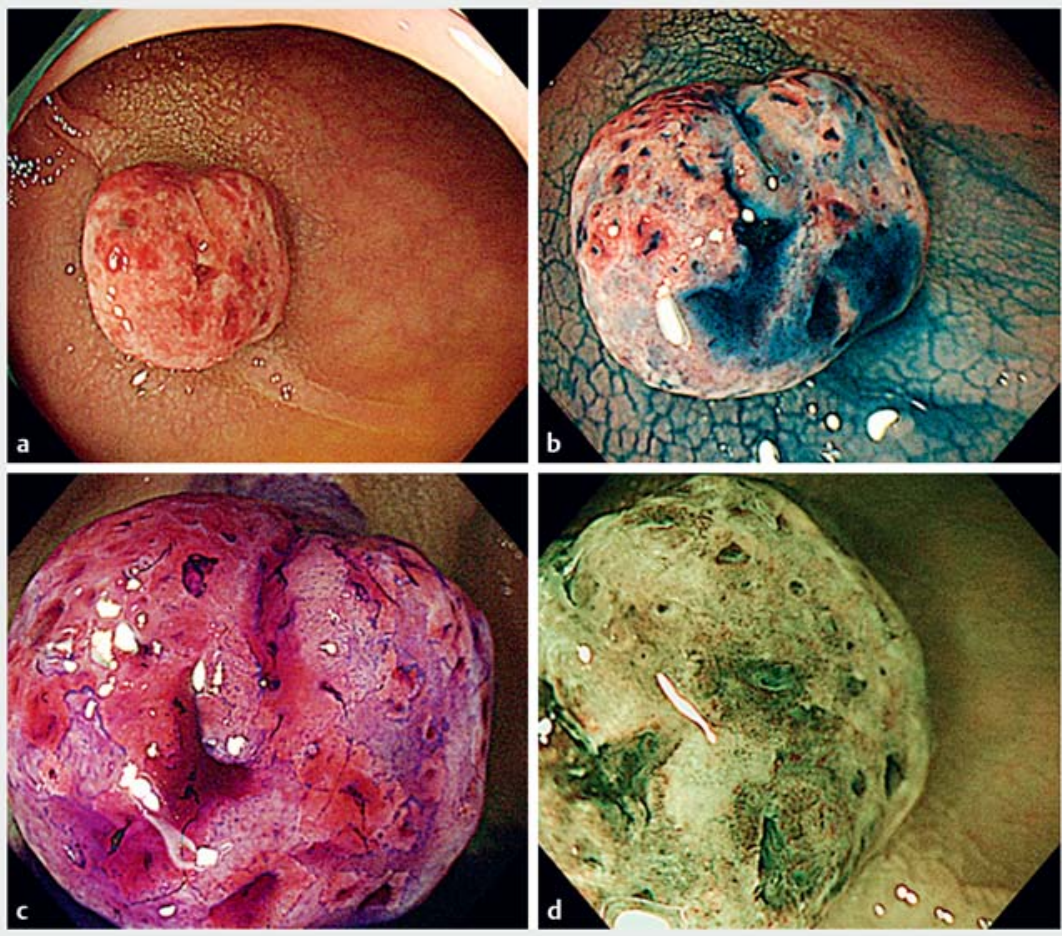

- Fig. 1 a White-light imaging: a reddish elevated lesion with erosion was seen. b, c Chromoendoscopy: $\mathbf{b}$ indigo carmine, $\mathbf{c}$ crystal violet; elongated and irregularly arranged ducts were revealed. $\mathbf{d}$ Magnifying endoscopy with narrow-band imaging: a dense pattern was observed close to the dilated ducts.
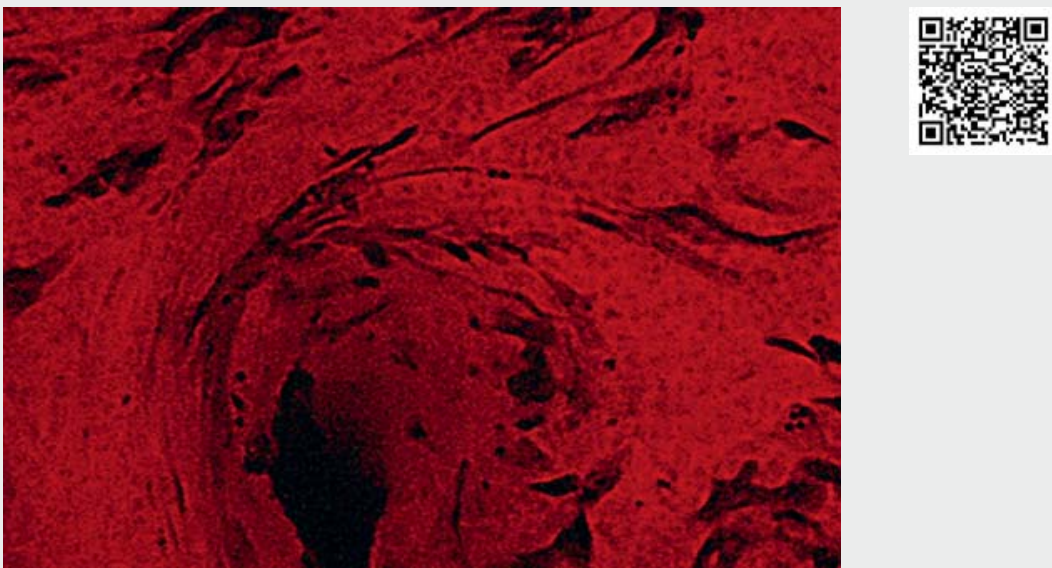

Video 1 Endocytoscopic images were obtained after staining with $1 \%$ methylene blue and $0.05 \%$ crystal violet. They showed regular elongated ducts with no apparent irregular nuclear findings around the ducts. 

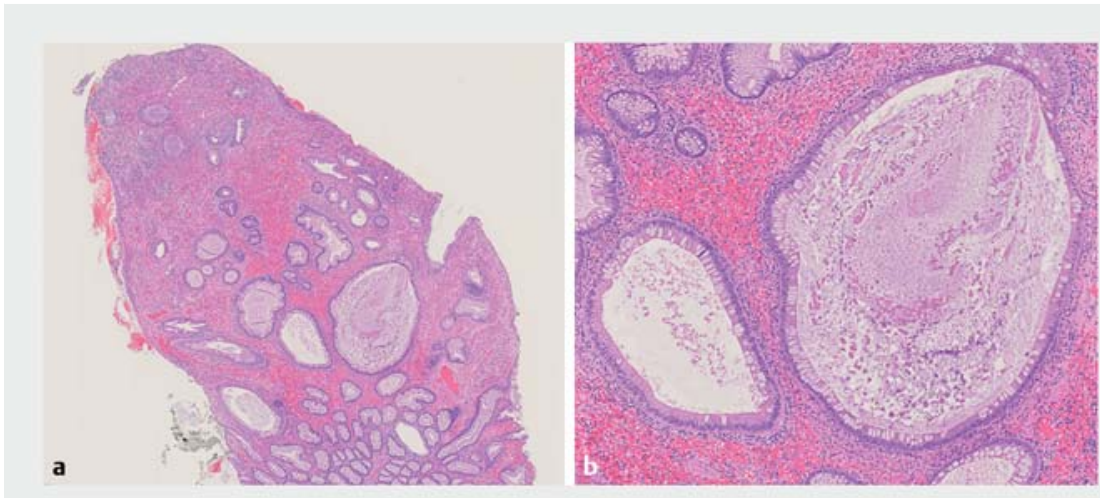

- Fig. 2 a, b Hematoxylin and eosin (H\&E) staining: the edematous open inflamed stroma and cystically dilated glands without atypia were seen (a orig. mag. $\times 10$, b orig. mag. $\times 40$ ).

In this case, the lesion was suspected at the referring hospital to be cancer, but our precise examination led us to suspect on the basis of magnifying endoscopy that it was a juvenile polyp, and the diagnosis was confirmed by endocytoscopy in real time. Subsequently, we were able to select appropriate treatment without a standard biopsy.

Endoscopy_UCTN_Code_CCL_1AD_2AC

\section{Acknowledgments}

This work was supported in part by the National Cancer Center Research and Development Fund (29-A-13) and AMED under Grant Number JP18hk0102034.

\section{Competing interests}

None
The authors

Kaizo Kagemoto ${ }^{1,3}$, Yutaka Saito ${ }^{1}$, Yasuhiko

Mizuguchi ${ }^{1}$, Taku Sakamoto ${ }^{1}$, Hirokazu

Taniguchi' ${ }^{2}$, Shigeki Sekine ${ }^{2}$, Tetsuji

Takayama $^{3}$

1 Endoscopy Division, National Cancer Center Hospital, Tokyo, Japan

2 Clinical Laboratory Division, National Cancer Center Hospital, Tokyo, Japan

3 Department of Gastroenterology and Oncology, Institute of Biomedical Sciences, Tokushima University Graduate School, Japan

\section{Corresponding author}

\section{Yutaka Saito, MD, PhD}

Endoscopy Division, National Cancer Center Hospital, 5-1-1 Tsukiji, Chuo-ku, Tokyo, 104-0045, Japan

ytsaito@ncc.go.jp

[1] Sasajima K, Kudo SE, Inoue H et al. Real-time in vivo virtual histology of colorectal lesions when using the endocytoscopy system. Gastrointest Endosc 2006; 63: 1010-1017

[2] Kudo SE, Wakamura K, Ikehara N et al. Diagnosis of colorectal lesions with a novel endocytoscopic classification - a pilot study. Endoscopy 2011; 43: 869-875

[3] Utsumi T, Sano Y, Iwatate M et al. Prospective real-time evaluation of diagnostic performance using endocytoscopy in differentiating neoplasia from nonneoplasia for colorectal diminutive polyps $(\leq 5 \mathrm{~mm})$. World J Gastrointest Oncol 2018; 10: 96102

[4] Mori Y, Kudo SE, Misawa M et al. Real-time use of artificial intelligence in identification of diminutive polyps during colonoscopy: a prospective study. Intern Med 2018; 169 : 357-366

[5] Hirotani A, Sakai E, Nakajima A et al. Endoscopic findings of atypical juvenile colonic polyps. Gastrointest Endosc 2016; 83: 476477

\section{Bibliography}

DOI https://doi.org/10.1055/a-1028-6973

Published online: 7.11.2019

Endoscopy 2020; 52: E142-E143

(c) Georg Thieme Verlag KG

Stuttgart · New York

ISSN 0013-726X

\section{ENDOSCOPY E-VIDEOS \\ https://eref.thieme.de/e-videos}

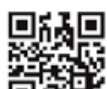
Endoscopy E-Videos is a free access online section, reporting on interesting cases and new techniques in gastroenterological endoscopy. All papers include a high quality video and all contributions are freely accessible online.

This section has its own submission website at https://mc.manuscriptcentral.com/e-videos 